\title{
Activity-Dependent Palmitoylation Controls SynDIG1 Stability, Localization, and Function
}

\author{
Inderpreet Kaur, ${ }^{1}$ - Vladimir Yarov-Yarovoy, ${ }^{2}$ Lyndsey M. Kirk, ${ }^{1}$ Kristopher E. Plambeck, ${ }^{1}$ ㅌden V. Barragan, ${ }^{1}$ \\ Eric S. Ontiveros, ${ }^{1}$ and ${ }^{\circledR E}$ Elva Díaz ${ }^{1}$ \\ Departments of ${ }^{1}$ Pharmacology, and ${ }^{2}$ Physiology and Membrane Biology, University of California Davis School of Medicine, Davis, California 95616
}

\begin{abstract}
Synapses are specialized contacts between neurons. Synapse differentiation-induced gene I (SynDIG1) plays a critical role during synapse development to regulate AMPA receptor (AMPAR) and PSD-95 content at excitatory synapses. Palmitoylation regulates the localization and function of many synaptic proteins, including AMPARs and PSD-95. Here we show that SynDIG1 is palmitoylated, and investigate the effects of palmitoylation on SynDIG1 stability and localization. Structural modeling of SynDIG1 suggests that the membrane-associated region forms a three-helical bundle with two cysteine residues located at positions 191 and 192 in the juxta-transmembrane region exposed to the cytoplasm. Site-directed mutagenesis reveals that C191 and C192 are palmitoylated in heterologous cells and positively regulates dendritic targeting in neurons. Like PSD-95, activity blockade in a rat hippocampal slice culture increases SynDIG1 palmitoylation, which is consistent with our prior demonstration that SynDIG1 localization at synapses increases upon activity blockade. These data demonstrate that palmitoylation of SynDIG1 is regulated by neuronal activity, and plays a critical role in regulating its stability and subcellular localization, and thereby its function.
\end{abstract}

Key words: excitatory synapse; palmitoylation; PSD-95; SynDIG1

Significance Statement

Palmitoylation is a reversible post-translation modification that has recently been recognized as playing a critical role in the localization and function of many synaptic proteins. Here we show that activity-dependent palmitoylation of the atypical AMPA receptor auxiliary transmembrane protein SynDIG1 regulates its stability and localization at synapses to regulate function and synaptic strength.

\section{Introduction}

Activity of excitatory synapses is dictated by the number and functional properties of AMPA-type glutamate receptors (AMPARs), which cycle in and out of synapses in an activitydependent manner (Malinow and Malenka, 2002; Bredt and Nicoll, 2003; Yokoi et al., 2012). We identified a novel type II transmembrane protein, synapse differentiation-induced gene I (SynDIG1), that regulates excitatory synapse number and strength in hippocampal neurons (Kalashnikova et al., 2010). SynDIG1 associates with AMPARs in heterologous cells and

\footnotetext{
Received Nov. 27, 2014; revised June 6, 2016; accepted June 10, 2016.

Author contributions: I.K., V.Y.Y., and E.D. designed research; I.K., V.Y.-Y., L.M.K., K.E.P., E.V.B., and E.S.O. performed research; I.K., V.Y.-Y., L.M.K., K.E.P., E.V.B., and E.D. analyzed data; I.K., V.Y.-Y., and E.D. wrote the paper.

This work was supported by funds to E.D. from the National Institutes of Health Director's New Innovator Award Program (Grant DP2-0D-006479-01), the National Science Foundation (Grant 1322302), and the Whitehall Foundation (Grant 2015-05-106). We thank Julie Culp and Karen Zito for providing rat hippocampal slice cultures, and members of the Diaz laboratory for helpful and insightful input on this project.

The authors declare no competing financial interests.

Correspondence should be addressed to Elva Díaz, Department of Pharmacology, UC Davis School of Medicine, 451 Health Sciences Drive, 3503 GBSF, Davis, CA 95616. E-mail: ediaz@ucdavis.edu.

DOI:10.1523/JNEUROSCI.4859-14.2016

Copyright $\odot 2016$ the authors $\quad 0270-6474 / 16 / 367562-07 \$ 15.00 / 0$
}

brain lysates, and requires its C-terminal region for association (Kalashnikova et al., 2010). In contrast to other AMPAR auxiliary subunits, SynDIG1 overexpression in hippocampal slice culture does not alter surface expression or biophysical properties of AMPARs (Lovero et al., 2013), suggesting that SynDIG1 is an atypical accessory protein with a unique mechanism. The C-terminal portion of SynDIG1, the most conserved region across species as well as other family members (SynDIG2-4), encodes the membrane-associated domain, which is important for its ability to promote synapse development (Kalashnikova et al., 2010). Additionally, SynDIG1 accumulates at synapses upon activity blockade by tetrodotoxin (TTX), increasing the Syn DIG1 spine/shaft ratio as determined by immunocytochemistry (Kalashnikova et al., 2010).

S-Palmitoylation is a reversible post-translational modification that regulates membrane association, trafficking, and protein-protein interactions (Blaskovic et al., 2013). Addition of the 16 carbon palmitate moiety to cysteine residues via thioester linkage is catalyzed by palmitoyl acyltransferases (PATs). Many synaptic proteins are palmitoylated in an activity-dependent manner (Kang et al., 2008; Fukata and Fukata, 2010; Thomas and 
Huganir, 2013). For example, activity-dependent palmitoylation of PSD-95 regulates its localization at synapses (El-Husseini Ael et al., 2002). AMPAR palmitoylation regulates trafficking in a subunit-specific manner, and depalmitoylation is mediated by receptor activity (Hayashi et al., 2005).

In the present study, structural modeling predicts that Syn DIG1 contains two conserved cysteine residues in its juxtatransmembrane region at positions 191 and 192. A distant relative of the SynDIG family, IFITM3, has been shown to be palmitoylated at cysteines located at similar positions (Yount et al., 2010). We demonstrate that SynDIG1 is palmitoylated at C191 and C192. Mutation of these residues prevents palmitoylation and negatively impacts subcellular localization and stability, and therefore function. Furthermore, we demonstrate that SynDIG1 palmitoylation is regulated by activity, suggesting a mechanism for SynDIG1 relocalization to synapses upon activity blockade, which has been demonstrated previously (Kalashnikova et al., 2010). Together, these data imply that activity-dependent palmitoylation is a mechanism that has been widely used to control synapse strength via alterations in synaptic protein localization.

\section{Materials and Methods}

Animals. Sprague Dawley timed pregnant rats were purchased from Harlan/Envigo or Charles River. C57BL/6 mice were bred in the animal facility at the University of California (UC) Davis. Animals of either sex were used in all experiments. The use and maintenance of animals were performed according to guidelines set forth by UC Davis, the National Institutes of Health, and the Association for Assessment and Accreditation of Laboratory Animal Care.

Antibodies. The following antibodies were used: mouse anti-SynDIG1 [NeuroMab; immunoblot (IB), 1:1000; immunocytochemistry (ICC), 1:100]; rat anti-HA (Roche; IB, 1:2000; ICC, 1:200); mouse anti-GM130 (BD Transduction Laboratories; ICC, 1:500); rabbit anti-EEA1 (catalog \#2900, Abcam; ICC, 1:200); rabbit anti-calreticulin (catalog \#2907, Abcam; ICC, 1:600); mouse anti-PSD-95 (NeuroMab; IB, 1:5000; ICC, 1:200); guinea pig anti-VGluT1 (Millipore; ICC, 1:500); mouse anti- $\beta$ tubulin (BD Biosciences; IB, 1:10,000); rabbit anti-microtubuleassociated protein 2 (MAP2; Sigma-Aldrich; ICC, 1:500); Alexa Fluor 488- and 594-conjugated antibodies (Invitrogen; ICC, 1:200); DyLight 649-, Cy3-, or Cy5-conjugated antibodies (Jackson ImmunoResearch; ICC, 1:200); and HRP-conjugated antibodies (Invitrogen; IB, 1:50,000).

Acyl-biotin exchange assay. Palmitoylation was detected using the acyl-biotin exchange (ABE) assay as published (Wan et al., 2007). Briefly, $10 \mathrm{~mm} N$-ethylmaleimide blocks free thiols, $0.7 \mathrm{~m}$ hydroxylamine (HAM) cleaves Cys-palmitoyl thioester linkages, and free thiols are labeled using $1 \mathrm{~mm}$ thiol-reactive biotinylation reagent (HPDPbiotin) followed by NeutrAvidin precipitation. Palmitoylated and total levels (10\% of input) of SynDIG1 and PSD-95 were detected by immunoblotting.

Constructs. For site-directed mutagenesis, primers were designed using the QuickChange program (Agilent Technologies) to change codons for cysteine at positions 191 and 192 to alanine or serine with wild-type (WT) SynDIG1 as a template. The first reaction used mutagenic forward primer and WT reverse primer with EcoRI restriction site to introduce the mutation while amplifying an $\sim 200$ bp product. WT forward primer with KpnI restriction site and PCR product from the first round ("mega-primer") were then used to amplify full-length mutant SynDIG1 in the second round of PCR and were cloned into pHM6 vector to introduce an N-terminally encoded HA tag. All constructs were verified by sequencing.

Culture of COS cells and hippocampal neurons/slices. COS cells were seeded in six-well plates $\left(3 \times 10^{5}\right.$ cells/well $) 24 \mathrm{~h}$ before transfection. For immunocytochemistry, cells were seeded on coverslips coated with 25 $\mu \mathrm{g} / \mathrm{ml}$ poly-L-lysine in $0.1 \mathrm{M}$ borate buffer at $37^{\circ} \mathrm{C}$ for $1 \mathrm{~h}$ and washed with water. Cells were cultured at $37^{\circ} \mathrm{C}$ with $5 \% \mathrm{CO}_{2}$ in $\mathrm{COS}$ media (DMEM with GlutaMAX, 10\% fetal bovine serum, $100 \mu \mathrm{g} / \mathrm{ml}$ penicillin/streptomycin). Cells were transfected in Opti-MEM with a 2:3 ratio of DNA $(2 \mu \mathrm{g})$ to Lipofectamine 2000 reagent $(3 \mu \mathrm{l})$. After $3-4 \mathrm{~h}$, cells were washed with $1 \times$ PBS and maintained in COS media for $24 \mathrm{~h}$. All culture media and reagents were from Life Technologies. For Brefeldin A (BFA) experiments, cells were treated with BFA $(5 \mu \mathrm{g} / \mathrm{ml})$ or vehicle (methanol) for $30 \mathrm{~min}$ before immunocytochemistry. For immunoblotting, cells were washed with $1 \times \mathrm{PBS}$ and harvested in lysis buffer [ $150 \mathrm{~mm} \mathrm{NaCl}, 50$ mм Tris $\mathrm{pH} 7.4,1 \%$ Triton X-100, and protease inhibitor cocktail (23.4 $\mu \mathrm{M}$ leupeptin, $6.1 \mu \mathrm{M}$ aprotinin, $14.5 \mu \mathrm{M}$ pepstatin $\mathrm{A}$, and $0.1 \mathrm{~mm}$ phenylmethylsulfonyl fluoride)]. Lysates were incubated on a rotator at $4^{\circ} \mathrm{C}$ for $30 \mathrm{~min}$ and centrifuged at $12,000 \times g$ for $10 \mathrm{~min}$. Supernatants were collected, and protein concentrations were measured using the BCA assay. For cycloheximide (CHX) experiments, transfected cells were treated with $100 \mu \mathrm{M}$ CHX for up to $24 \mathrm{~h}$ before harvesting.

Dissociated rat hippocampal neurons were prepared as described previously (Kalashnikova et al., 2010). Neurons were transfected at $4-5 \mathrm{~d}$ in vitro (DIV) by calcium phosphate precipitation and maintained for up to 8-11 DIV. For some experiments, neurons were treated at 10 DIV with $2 \mu \mathrm{M}$ TTX or vehicle [distilled water $\left(\mathrm{dH}_{2} \mathrm{O}\right)$ ] for $2 \mathrm{~d}$ before immunocytochemistry. For some experiments, neurons were treated at $11 \mathrm{DIV}$ for $4 \mathrm{~h}$ with $50 \mu \mathrm{M}$ 2-bromopalmitate (2-BP) or vehicle (DMSO) before immunocytochemistry.

Organotypic hippocampal slice cultures were prepared from P6 to P7 Sprague Dawley rats, as previously described (Hill and Zito, 2013). Slice cultures (7 DIV) were treated with vehicle $\left(\mathrm{dH}_{2} \mathrm{O}\right)$ or $2 \mu \mathrm{M}$ TTX for $16 \mathrm{~h}$ at $37^{\circ} \mathrm{C}$ and subjected to $\mathrm{ABE}$ assay.

Immunocytochemistry. Cells were fixed in $4 \%$ paraformaldehyde in $1 \times$ PBS for 10 min at room temperature (RT). Coverslips were rinsed in $1 \times$ PBS, permeabilized for 10 min at RT with $0.1 \%$ Triton X-100 in $1 \times$ PBS, and blocked with $5 \%$ nonfat milk in $1 \times$ PBS for 30 min. After primary antibody incubation overnight at $4^{\circ} \mathrm{C}$ and washes in $1 \times \mathrm{PBS}(3 \times 10$ $\mathrm{min})$, coverslips were incubated with secondary antibodies diluted in blocking solution for $1 \mathrm{~h}$ at RT. Following washes in $1 \times$ PBS, coverslips were mounted on microscope slides with Fluoromount-G media (SouthernBiotech).

Single images were acquired using a Zeiss LSM 710 confocal microscope under a $63 \times / 1.4$ oil-immersion objective or an Olympus Fluoview 1000 confocal microscope under a $40 \times$ objective lens with identical settings for laser power, photomultiplier gain, and digital offset within each dataset. Pinhole (1 A.U.) and resolution $(1132 \times 1132$ pixels $)$ were constant for all images. To quantitatively examine SynDIG1 localization, images were imported in Zeiss AxioVision version 4.4 or ImageJ software. Thresholds were established using a subset of images from each dataset so that all puncta were included, and the average threshold applied to the entire dataset. The cell body was excluded from analysis. Synapse number was measured as PSD-95 and VGluT1 colocalized puncta. The percentage of synapses containing SynDIG1 was calculated as synapses containing SynDIG1/total synapses $* 100$. To calculate the fold enrichment, the percentage of SynDIG1 with each subcellular marker was determined as follows: (number of SynDIG1 puncta colocalized with marker) $/[$ (number of SynDIG1 puncta) + (number of marker puncta) $] \times 100$. Values were normalized to WT SynDIG1. For figure preparation, signals were adjusted for all panels within a figure by using equal linear adjustments of levels in Photoshop (Adobe Systems). Data were collected from at least two independent experiments. Graphs and statistical analyses were generated in GraphPad Prism software, and data are represented as the mean \pm SEM. Statistical significance was assessed by paired Student's $t$ test or one-way ANOVA and defined as follows: ${ }^{\star} p<0.05,{ }^{* *} p<0.01$, and ${ }^{* * *} p<0.001$.

Structural modeling of SynDIG1. De novo and full atom modeling of the membrane-spanning region (residues P178-L258) of mouse SynDIG1 was performed using RosettaMP (Yarov-Yarovoy et al., 2006, 2012; Barth et al., 2007). Octopus server (Viklund and Elofsson, 2008) predicted two membrane-spanning regions formed by residues from G183 to L203 and from L229 to I249. However, based on the secondary structure prediction by JNET (Cuff and Barton, 1999) and PROF (Ouali and King, 2000; Rost, 2001), the second membrane-spanning region was predicted to have a helix break formed by residues G237, T238, and G239, and suggested the formation of a membrane re-entrant region in the structure. Therefore, only the first membrane-spanning region was constrained to span the membrane during de novo protein folding (Yarov-Yarovoy et al., 
2006). The second membrane-spanning region was free to sample different topologies. A total of 10,000 low-resolution de novo models were generated followed by model clustering (Yarov-Yarovoy et al., 2006). A total of 10,000 full-atom models were generated starting from 20 low-resolution models representing top 20 clusters (Barth et al., 2007; Yarov-Yarovoy et al., 2012). The lowest energy and top cluster full-atom models were analyzed visually. The second top cluster and several of the lowest energy models showed the membrane re-entrant region within the predicted second membranespanning region. A Ramachandran plot was generated using the Ramachandran plot function of UCSF Chimera (Pettersen et al., 2004) with probability contours based on a reference set of high-resolution proteins (Lovell et al., 2003).

\section{Results}

SynDIG1 is a type II membrane protein with a single transmembrane domain and a second hydrophobic segment that does not span the membrane (Kalashnikova et al., 2010). Structural modeling of this region of mouse SynDIG1 (residues P178L258) predicts a three-helical bundle configuration composed of the transmembrane helix and two independent helices from the hydrophobic segment (Fig. $1 A$, top). Typically, palmitoylation of transmembr-

ane proteins occurs on cysteine residues located near the transmembrane region (Blaskovic et al., 2013). The space-filling model reveals two cysteine residues (C191 and C192) in the juxta-transmembrane region of SynDIG1 that face the cytoplasm (Fig. 1A, bottom). However, a magnified view of the region suggests that the C191 thiol group is hindered by side chains of neighboring amino acids (Fig. $1 B$ ), while the $\mathrm{C} 192$ thiol group is available for modification (Fig. 1C).

To investigate whether endogenous SynDIG1 is palmitoylated, hippocampi from 1-month-old mice were subjected to ABE assay and probed with anti-SynDIG1 antibody (Fig. 2A). We detected SynDIG1 immunoreactivity in the HAM-treated fraction but not in the control, untreated fraction (Fig. $2 A$ ), indicating that a portion of SynDIG1 is palmitoylated in vivo.

We replaced C191 or C192 with alanine individually (C191A, C192A) or in combination (C191,192A) or with serine (C191,192S) using site-directed mutagenesis with HAtagged SynDIG1 as a template (Kalashnikova et al., 2010). Initial experiments indicated that C192A, C191,192A, and C191,192S mutants showed reduced expression in COS cells compared with WT (data not shown), but this difference could be overcome by transfecting higher quantities of plasmid DNA. To assess palmitoylation, constructs were transfected into COS cells and subjected to ABE assay (Fig. 2B). C191A did not affect SynDIG1 palmitoylation, whereas palmitoylation was abolished for C191,192A and C191,192S mu-
B
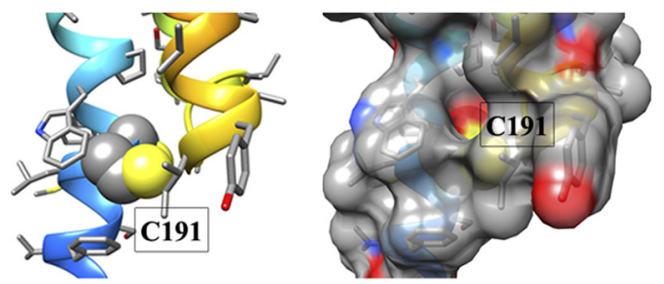

C
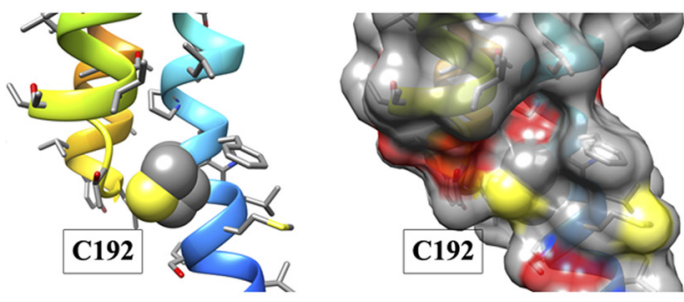

D

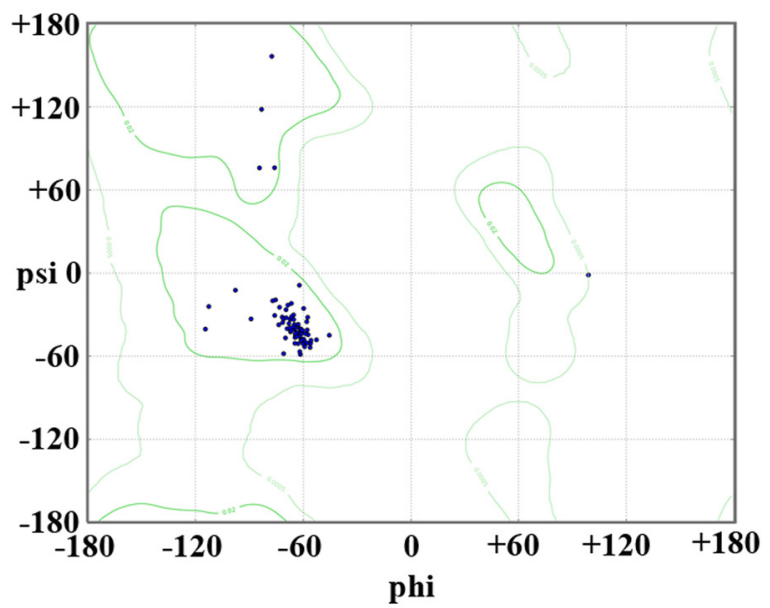

Figure 1. Structural model of mouse SynDIG1 membrane-associated region. $\boldsymbol{A}$, Transmembrane view of ribbon (top) and surface (bottom) representation of the Rosetta model. Membrane segments colored by rainbow color scheme-from the (blue) to the C-terminal (red) region. Side chains of $\mathrm{N}$ - and C-terminal residues and residues within the 1236 - G239 I00p Carbon, nitrogen, oxygen, and sulfur atoms are colored gray, blue, red, and yellow, respectively. $\boldsymbol{B}$, Zoomed-in view of structural model in $\boldsymbol{A}$. Each residue is a dot in the graph of $\varphi$ vs $\psi$ backbone angles. Probability contours based on a reference set of high-resolution proteins are shown on the plot as green lines.

tants and were substantially reduced for C192A (Fig. 2B), suggesting that both cysteines are palmitoylated, with C192 being the principal site.

As mentioned, SynDIG1 levels were reduced by palmitoylation-deficient mutations compared with WT, indicating an effect on stability. To test this possibility, cells transfected with SynDIG1 constructs were treated with CHX to inhibit protein synthesis, and lysates were collected at $0,3,8$ and $24 \mathrm{~h}$ post-CHX treatment. As shown in Figure 2, C and D, C191,192A expression was dramatically reduced within $8 \mathrm{~h}$ of CHX treatment, while WT expression was stable. To calculate the half-life of WT and C191,192A in COS cells, three independent experiments were performed. The slope of the line for WT and C191,192A expression was calculated with the equation $y=m x+b$, where $y$ is the percentage of SynDIG1 detected, $m$ is the slope of the line, $x$ is the number of hours post-CHX treatment, and $b$ is 100 . The half-life 
A

B

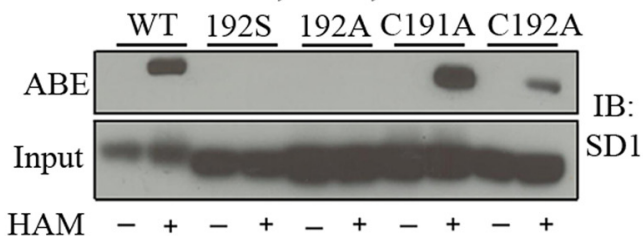

C
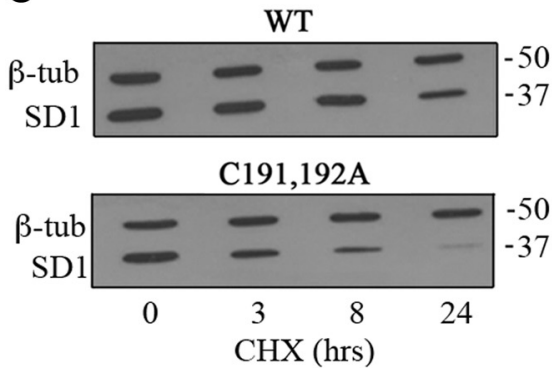

E
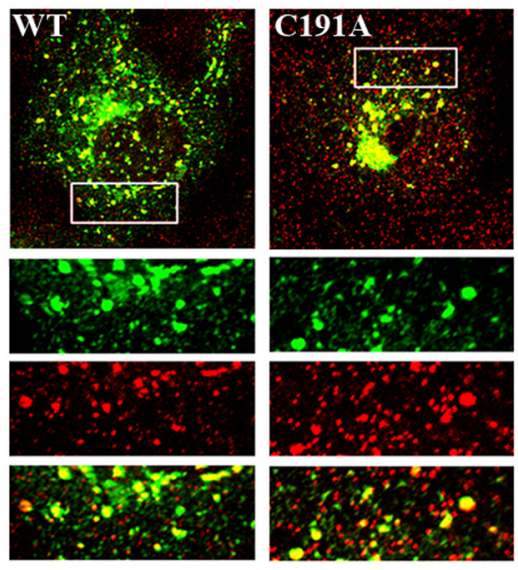

D

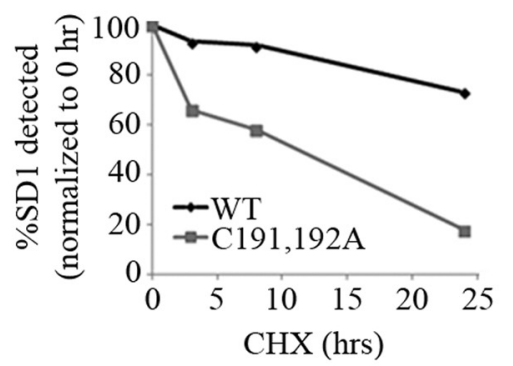

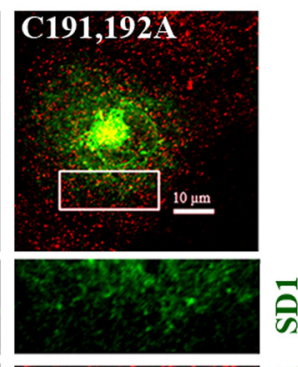
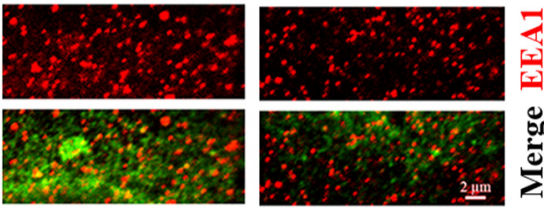

$\mathbf{F}$

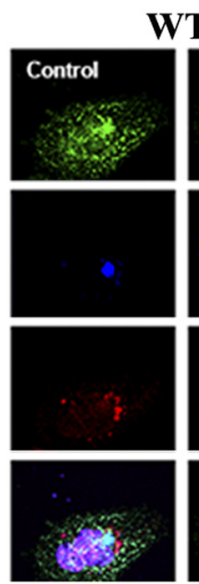

WT
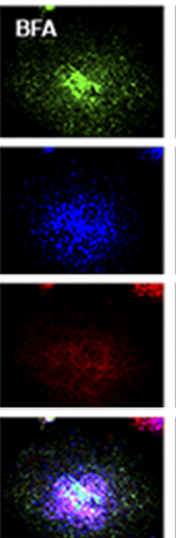

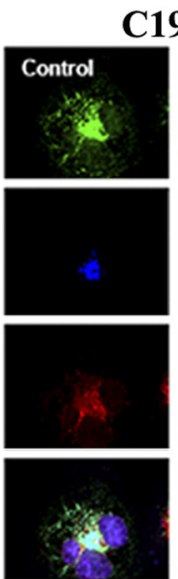

C191A

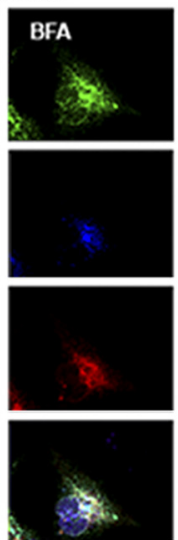

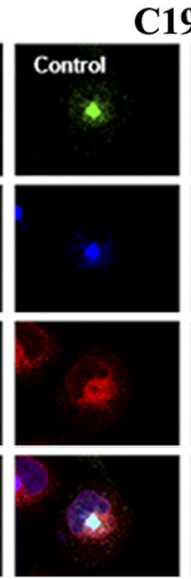

C192A

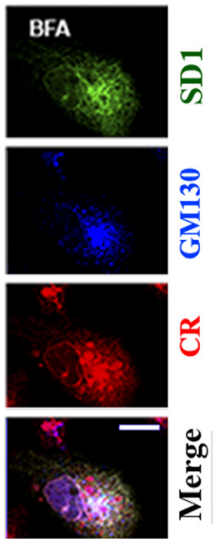

Figure 2. SynDIG1 palmitoylation is required for clustering and stability in COS cells. $\boldsymbol{A}$, Hippocampi from 1-month-old mice were lysed and subjected to ABE assay. Palmitoylated (ABE) SynDIG1 and total protein represented by $10 \%$ of the input sample in the presence or absence of HAM were measured by immunobloting with anti-SynDIG1 antibodies (SD1). $\boldsymbol{B}$, Identification of SynDIG1 palmitoylation sites. COS cells were transfected with HA-tagged WT or mutant forms of SynDIG1. After $24 \mathrm{~h}$, cells were lysed and subjected to ABE assay. Palmitoylated (ABE) SynDIG1 and total protein represented by $10 \%$ of the input sample in the presence or absence of HAM were measured by immunoblotting with anti-SD1 antibodies. C, Stability of WT and palmitoylationdeficient SynDIG1 mutant C191,192A in COS cells was investigated by treatment with $100 \mu \mathrm{g} / \mathrm{ml}$ CHX for indicated times. Immunoblotting for $\beta$-tubulin ( $\beta$-tub) served as a loading control. $\boldsymbol{D}$, Graph depicts the percentage of SynDIG1 detected by immunoblotting from lysates isolated after $\mathrm{CHX}$ treatment normalized to samples at $0 \mathrm{~h}$. $\boldsymbol{E}, \mathrm{SynDIG1}$ clustering in heterologous cells requires palmitoylation of 192 . COS cells were transfected with WT or mutant forms of SynDIG1, fixed after $24 \mathrm{~h}$, and labeled with anti-SD1 (green) and anti-EEA1 (red) antibodies. Bottom, Zoomed-in image of the boxed region in the top panel. Scale bars: $\boldsymbol{E}, 10$ $\mu \mathrm{m}$; inset, $2 \mu \mathrm{m}$. $\boldsymbol{F}$, SynDIG1 lacking palmitoylation is retained in the secretory pathway. COS cells were transfected with

was estimated by solving the equation for $y=50 \%$ SynDIG1 detected, where the slope of the line was $m=-1.68$ for WT and $m=-3.17$ for C191,192A. Accordingly, the half-lives for WT and C191, 192A in COS cells were estimated to be 30 and $16 \mathrm{~h}$, respectively.

To determine whether palmitoylation state alters localization, the distribution of SynDIG1 was determined by immunocytochemistry. Compared with WT, the distribution of C192A and C191,192A mutants was drastically altered, while C191A was unchanged (Fig. 2E). WT and C191A were localized in clusters that overlap with the endosomal marker EEA1 (Fig. 2E), which is consistent with our published study (Kalashnikova et al., 2010). In contrast, C192A and C191,192A did not overlap with EEA1 clusters, suggesting retention in the secretory pathway. Indeed, treatment with BFA to disrupt endoplasmic reticulum (ER)-to-Golgi transport followed by immunostaining with markers for ER (calreticulin) and Golgi apparatus (GM130; Fig. 2F) are consistent with this interpretation.

To compare the neuronal distribution of palmitoylated and nonpalmitoylated SynDIG1, we overexpressed WT and mutant SynDIG1 in dissociated rat hippocampal neurons, fixed and stained with anti-HA, anti-EEA1, and anti-GM130 antibodies (Fig. 3A). While WT and C191A immunolabeling was clustered and extended far into distal dendrites, C192A was restricted to the cell soma and proximal dendrites. Indeed, relative to WT and C191A, C192A localization was enriched with GM130 while it was de-enriched with EEA1 (Fig. $3 B, C$ ), which is consistent with the results in COS cells. C192A localization was also enriched with calreticulin relative to WT and C191A (data not shown).

To determine whether palmitoylation influences endogenous SynDIG1, we treated dissociated hippocampal neurons with 2-BP or vehicle and stained them with antibodies against SynDIG1 or PSD95, VGluT1, and MAP2 (Fig. 3D). Pilot experiments defined the concentration and time of 2-BP treatment that did not affect neuronal health. Quantitative analysis of multiple images indicated that incubation with $50 \mu \mathrm{M} 2$ 2-BP decreased

\section{$\leftarrow$}

HA-tagged WT or mutant forms of SynDIG1. After $24 \mathrm{~h}$, cells were treated with BFA or vehicle (Control) for $30 \mathrm{~min}$; fixed; and labeled with anti-HA (green), anti-GM130 (blue), and anti-calreticulin (CR; red) antibodies. Nuclei are indicated by Hoechst stain (magenta) in the merged image. Scale bar, $5 \mu \mathrm{m}$. 
the area and integrated density (ID) of PSD-95 puncta at synapses, as expected; however, the area and ID of SynDIG1 puncta at synapses increased (Fig. $3 E, F$ ). Upon treatment, the SynDIG1 signal increased along dendrites that might reflect increased area and ID of SynDIG1 puncta at synapses. To address this contribution, we measured the spine/shaft ratio of SynDIG1 in a subset of images. The mean pixel intensity of the spine/shaft ratio was increased in 2-BP-treated neurons (vehicle, $0.707 \pm 0.024 ; n=4$ cells, 3 stretches, 278 spines; 2 -BP, $0.820 \pm 0.030 ; n=4$ cells, 3 stretches, 226 spines; $p=0.0014$ ).

To explore activity-dependent palmitoylation, we treated hippocampal slice cultures at 7 DIV with $2 \mu \mathrm{M}$ TTX for $16 \mathrm{~h}$, subjected lysates to ABE assay, and immunoblotted with anti-SynDIG1 and antiPSD-95 antibodies. The total levels of SynDIG1 and PSD-95 remained unchanged after TTX treatment; however, the palmitoylated fraction was increased (Fig. 4A).

Because C191A did not affect the localization of SynDIG1 in neurons (Fig. $3 A-C$ ) but does appear to be a site of palmitoylation (Fig. 2B), we tested whether C191 palmitoylation contributed to the activity-dependent redistribution of SynDIG1. Hippocampal neurons transfected with WT and C191A were treated with TTX for $48 \mathrm{~h}$, and SynDIG1-containing synapses measured. In vehicle-treated neurons, we observed a small but significant reduction in the percentage of synapses that contained C191A compared with WT (Fig. $4 B, C$ ). However, both WT and C191A distribution increased at synapses in the presence of TTX. One possibility is that the palmitoylation at both C192 and C191 contributes to SynDIG1 localization under basal conditions, while palmitoylation at C191 alone is not sufficient for the redistribution of SynDIG1 to synapses upon activity blockade. Indeed, C192A failed to redistribute to synapses upon activity blockade (data not shown). Furthermore, C191A overexpression increased synapse density compared with untransfected vehicle-treated neurons (Fig. 4D), although the magnitude was less than WT, likely due to a reduction in synaptic localization (Fig. 4C).

\section{Discussion}

SynDIG1 regulates synapse development via the modulation of synaptic AMPAR and PSD-95 content in dissociated hippocampal neurons (Kalashnikova et al., 2010). In contrast to other auxiliary subunits, SynDIG1 overex-
A
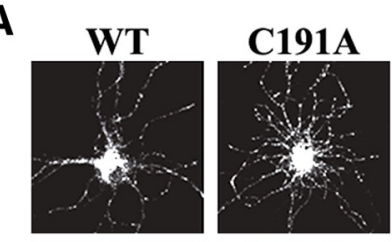

C192A
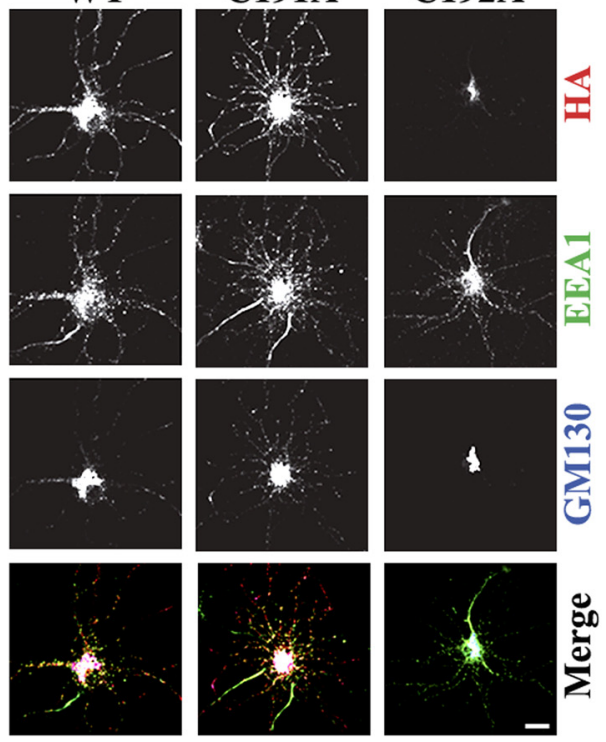

B

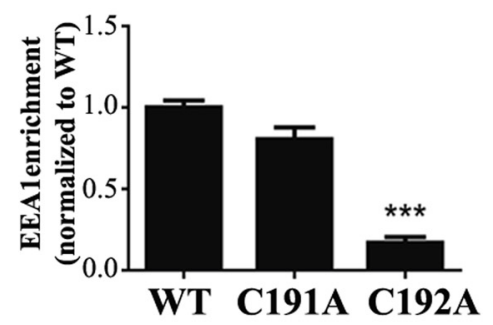

C

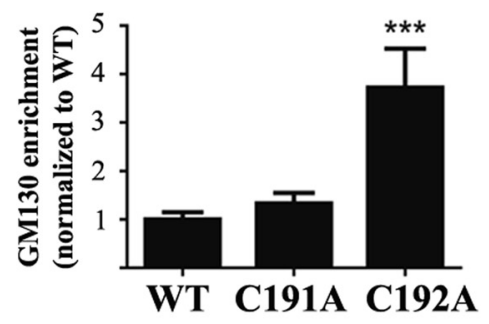

D
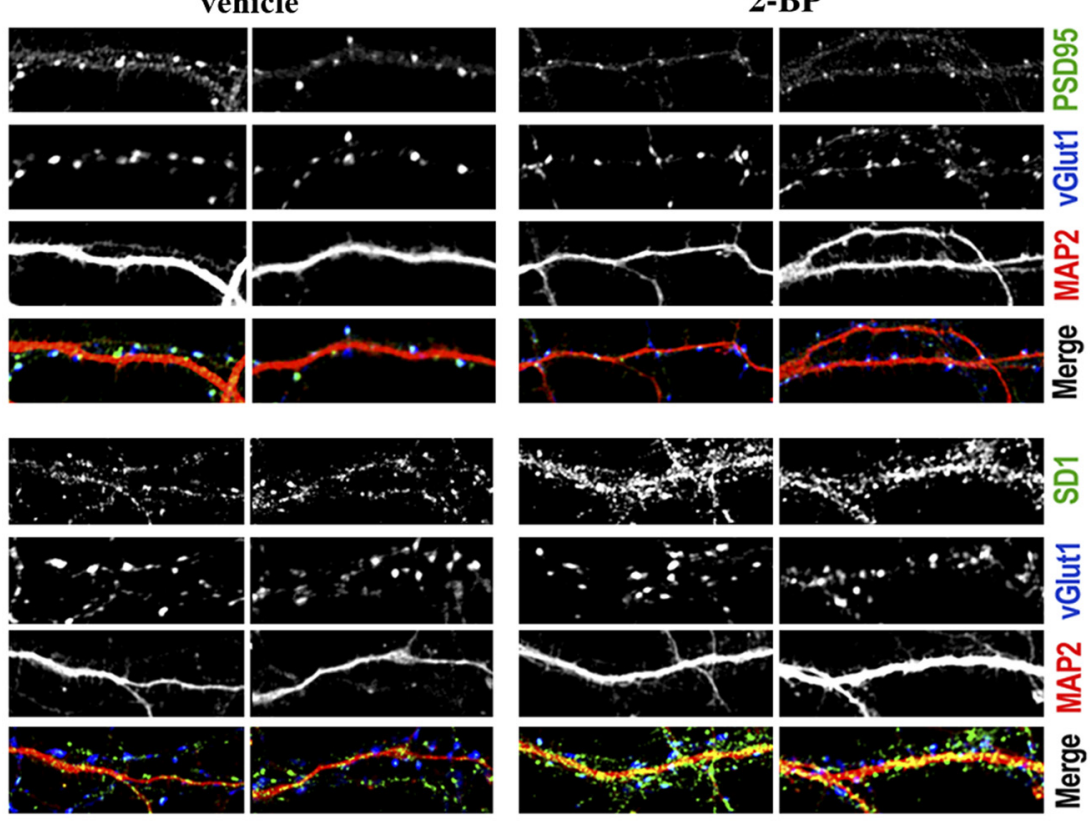

E
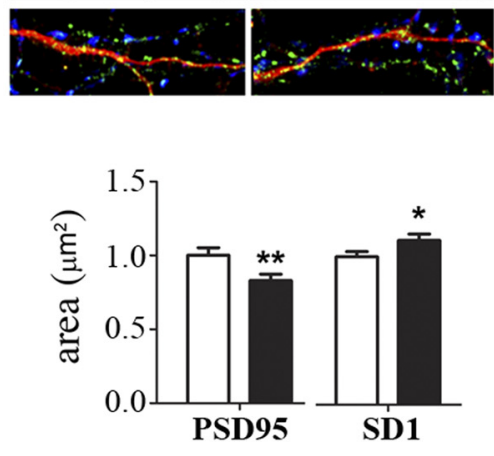

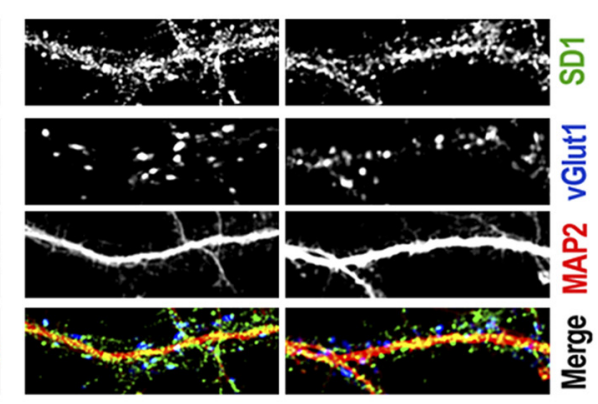

$\mathbf{F}$

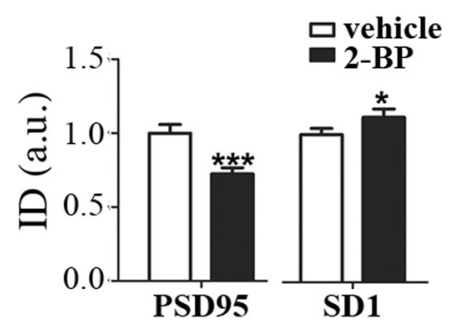

Figure 3. SynDIG1 localization in neurons requires (192 palmitoylation. $A$, Hippocampal neurons were transfected at 5 DIV with HA-tagged WT or mutant forms of SynDIG1, fixed at 9 DIV and immunostained with anti-HA (red), anti-EEA1 (green), and anti-GM130 (blue) antibodies. Scale bar, $20 \mu \mathrm{m}$. B, C, Graphs represent enrichment of SynDIG1 colocalization with subcellular markers. Similar results were obtained in two independent experiments, $n=10$ cells for each condition. Error bars, mean \pm SEM. ${ }^{* * *} p<0.001$. D, Representative stretches of hippocampal neurons treated at 11 DIV with vehicle (DMSO) or $50 \mu \mathrm{m}$ 2-BP for $4 \mathrm{~h}$; fixed; and stained with antibodies against PSD-95 or SynDIG1 (SD1), VGluT1, and MAP2. Scale bar, $10 \mu \mathrm{m} . E, F$, Graphs represent the puncta size and ID of PSD-95 and SD1 upon 2-BP treatment compared with vehicle. Data are the average of two independent experiments, $n=25$ cells for each condition. Error bars, mean $\pm S E M$. ${ }^{*} p<0.05$, ${ }^{* *} p<0.01,{ }^{* * *} p<0.001$. 

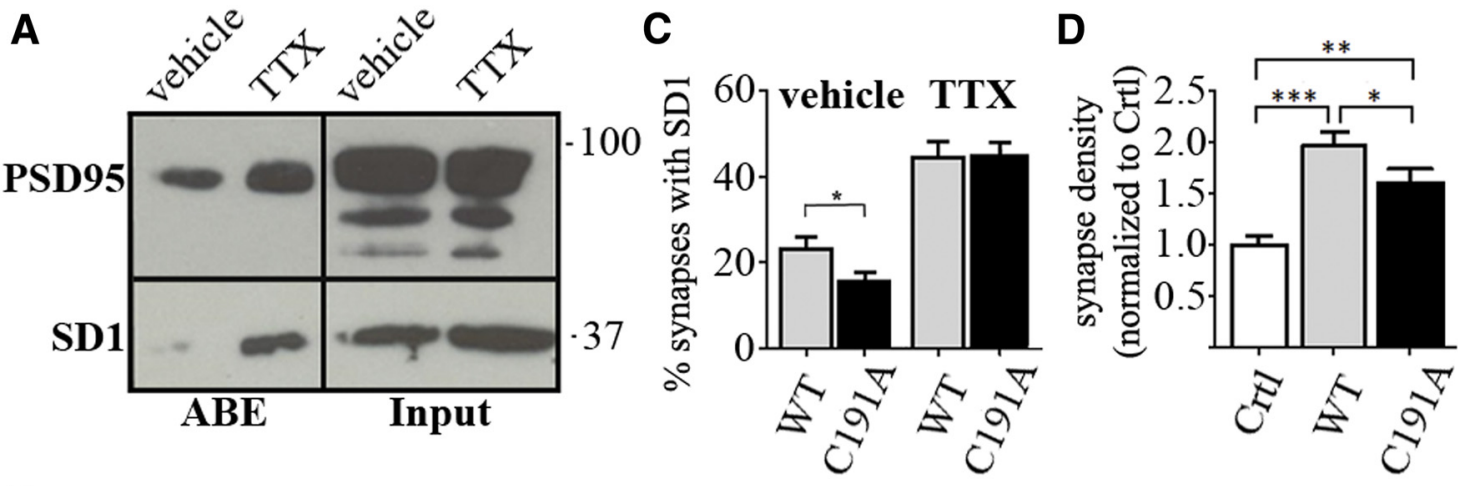

B

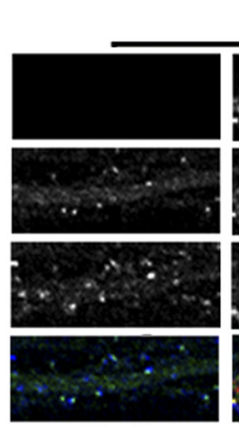

Crtl vehicle

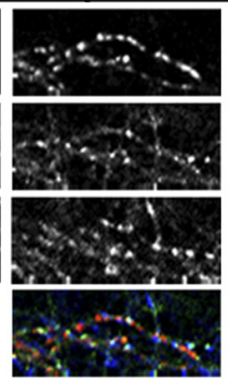

WT
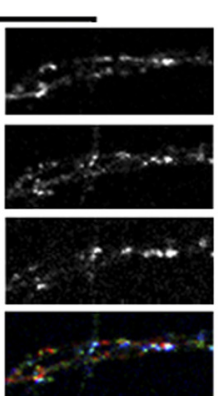

C191A

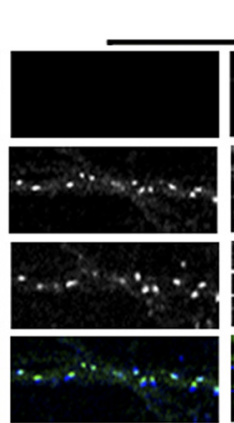

Crtl
TTX

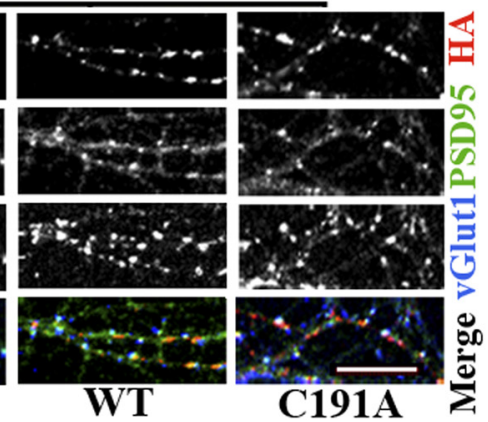

Figure 4. SynDIG1 palmitoylation is regulated by neuronal activity. $\boldsymbol{A}$, Blocking synaptic activity with TTX leads to increased SynDIG1 and PSD-95 palmitoylation. Hippocampal slice cultures were treated with $2 \mu \mathrm{m}$ TTX or vehicle for $16 \mathrm{~h}$ and subjected to ABE assay. B, Hippocampal neurons were transfected at 5 DIV with WT or C191A; treated with $2 \mu \mathrm{M}$ TTX or vehicle at 10 DIV; fixed at 12 DIV; and immunostained with antibodies against HA, PSD-95, and VGluT1. Synapses were defined as the colocalization of PSD-95 and VGluT1 clusters. Untransfected control (Crtl) neurons are shown for comparison. Scale bar, $10 \mu \mathrm{m}$. C, D, Graphs represent percentage synapses containing WT or C191A in vehicle- and TTX-treated neurons (C) or synapse density upon overexpression of WT or C191A compared with untransfected Crtl neurons in vehicle-treated samples (D). Data are the average of the following two independent experiments: vehicle: WT, $n=27$, C191A, $n=30 ;$ TTX: WT, $n=$ 30, C191A, $n=29$ (C); and Ctrl: $n=25 ;$ WT: $n=30 ;$ C191A: $n=30$ (D). Error bars, mean \pm SEM. ${ }^{*} p<0.05,{ }^{* *} p<0.01,{ }^{* * *} p<0.001$.

pression in hippocampal slice culture does not alter the surface expression or biophysical properties of AMPARs (Lovero et al., 2013), suggesting a unique mechanism. Determining the structure and function of SynDIG1 is, therefore, fundamental in elucidating the mechanism of the action of SynDIG1 at synapses. We report that SynDIG1 is palmitoylated at C191 and C192, and that this modification is important for its stability and localization. Furthermore, TTX treatment blocks increases in SynDIG1 palmitoylation in hippocampal slice culture. The results of this study reveal an important mechanism of SynDIG1 regulation in neurons and further appreciate the role of palmitoylation in synaptic homeostasis.

S-Palmitoylation of proteins is catalyzed by membrane-bound PAT enzymes that can be found at the ER, Golgi, endocytic vesicles, and plasma membrane. For example, palmitoylation of AMPARs near the channel pore causes its retention in the Golgi and requires depalmitoylation for forward trafficking to synapses (Hayashi et al., 2005). In contrast, palmitoylation of the cysteine residue located in the extreme $\mathrm{C}$ terminus of GluA2 regulates activity-induced internalization of AMPARs (Yang et al., 2009). Palmitoylation is also critical for AMPAR stability (Yang et al., 2009). An important finding of our study is that palmitoylation-deficient SynDIG1 has a shorter half-life than WT. Blocking palmitoylation with $16 \mathrm{~h}$ of treatment with $50 \mu \mathrm{M}$ 2-BP in hippocampal slice cultures indicated a reduction in both palmitoylated and total SynDIG1 (our unpublished observations). However, such treatment negatively impacts neuronal health because the incubation of dissociated hippocampal neuron cultures with 2-BP for $>4$ h caused massive cell death. Unexpectedly, the treatment of dissociated hippocampal neurons with
2-BP for $4 \mathrm{~h}$ increased the area and intensity of SynDIG1 puncta at synapses. In addition to PAT inhibition, 2-BP can also reduce deacylation by the inhibition of acyl-protein thioesterase enzymatic activities (Pedro et al., 2013), which might explain our unexpected observation. However, an alternative explanation is that SynDIG1 palmitoylation is critical for its endocytosis from the plasma membrane and/or retention in endosomes, which is consistent with the findings of previous studies demonstrating that the A-kinase anchoring protein 79/150 synaptic scaffold controls endosome retention independent of its role in plasma membrane targeting (Keith et al., 2012).

Altogether, our study provides mechanistic insight into SynDIG1 structure and function to demonstrate that its stability and subcellular localization are regulated by activitydependent palmitoylation.

\section{References}

Barth P, Schonbrun J, Baker D (2007) Toward high-resolution prediction and design of transmembrane helical protein structures. Proc Natl Acad Sci U S A 104:15682-15687. CrossRef Medline

Blaskovic S, Blanc M, van der Goot FG (2013) What does S-palmitoylation do to membrane proteins? FEBS J 280:2766-2774. CrossRef Medline

Bredt DS, Nicoll RA (2003) AMPA receptor trafficking at excitatory synapses. Neuron 40:361-379. CrossRef Medline

Cuff JA, Barton GJ (1999) Evaluation and improvement of multiple sequence methods for protein secondary structure prediction. Proteins 34: 508-519. CrossRef Medline

El-Husseini Ael-D, Schnell E, Dakoji S, Sweeney N, Zhou Q, Prange O, Gauthier-Campbell C, Aguilera-Moreno A, Nicoll RA, Bredt DS (2002) Synaptic strength regulated by palmitate cycling on PSD-95. Cell 108: 849-863. CrossRef Medline 
Fukata Y, Fukata M (2010) Protein palmitoylation in neuronal development and synaptic plasticity. Nat Rev Neurosci 11:161-175. CrossRef Medline

Hayashi T, Rumbaugh G, Huganir RL (2005) Differential regulation of AMPA receptor subunit trafficking by palmitoylation of two distinct sites. Neuron 47:709-723. CrossRef Medline

Hill TC, Zito K (2013) LTP-induced long-term stabilization of individual nascent dendritic spines. J Neurosci 33:678-686. CrossRef Medline

Kalashnikova E, Lorca RA, Kaur I, Barisone GA, Li B, Ishimaru T, Trimmer JS, Mohapatra DP, Díaz E (2010) SynDIG1: an activity-regulated, AMPA- receptor-interacting transmembrane protein that regulates excitatory synapse development. Neuron 65:80-93. CrossRef Medline

Kang R, Wan J, Arstikaitis P, Takahashi H, Huang K, Bailey AO, Thompson JX, Roth AF, Drisdel RC, Mastro R, Green WN, Yates JR 3rd, Davis NG, El-Husseini A (2008) Neural palmitoyl-proteomics reveals dynamic synaptic palmitoylation. Nature 456:904-909. CrossRef Medline

Keith DJ, Sanderson JL, Gibson ES, Woolfrey KM, Robertson HR, Olszewski K, Kang R, El-Husseini A, Dell'acqua ML (2012) Palmitoylation of A-kinase anchoring protein 79/150 regulates dendritic endosomal targeting and synaptic plasticity mechanisms. J Neurosci 32:7119-7136. CrossRef Medline

Lovell SC, Davis IW, Arendall WB 3rd, de Bakker PI, Word JM, Prisant MG, Richardson JS, Richardson DC (2003) Structure validation by Calpha geometry: phi,psi and Cbeta deviation. Proteins 50:437-450. CrossRef Medline

Lovero KL, Blankenship SM, Shi Y, Nicoll RA (2013) SynDIG1 promotes excitatory synaptogenesis independent of AMPA receptor trafficking and biophysical regulation. PLoS One 8:e66171. CrossRef Medline

Malinow R, Malenka RC (2002) AMPA receptor trafficking and synaptic plasticity. Annu Rev Neurosci 25:103-126. CrossRef Medline

Ouali M, King RD (2000) Cascaded multiple classifiers for secondary structure prediction. Protein Sci 9:1162-1176. CrossRef Medline

Pedro MP, Vilcaes AA, Tomatis VM, Oliveira RG, Gomez GA, Daniotti JL (2013) 2-Bromopalmitate reduces protein deacylation by inhibition of acyl-protein thioesterase enzymatic activities. PLoS One 8:e75232. CrossRef Medline

Pettersen EF, Goddard TD, Huang CC, Couch GS, Greenblatt DM, Meng EC, Ferrin TE (2004) UCSF Chimera-a visualization system for exploratory research and analysis. J Comput Chem 25:1605-1612. CrossRef Medline

Rost B (2001) Review: protein secondary structure prediction continues to rise. J Struct Biol 134:204-218. CrossRef Medline

Thomas GM, Huganir RL (2013) Palmitoylation-dependent regulation of glutamate receptors and their PDZ domain-containing partners. Biochem Soc Trans 41:72-78. CrossRef Medline

Viklund H, Elofsson A (2008) OCTOPUS: improving topology prediction by two-track ANN-based preference scores and an extended topological grammar. Bioinformatics 24:1662-1668. CrossRef Medline

Wan J, Roth AF, Bailey AO, Davis NG (2007) Palmitoylated proteins: purification and identification. Nat Protocols 2:1573-1584. CrossRef Medline

Yang G, Xiong W, Kojic L, Cynader MS (2009) Subunit-selective palmitoylation regulates the intracellular trafficking of AMPA receptor. Eur J Neurosci 30:35-46. CrossRef Medline

Yarov-Yarovoy V, Schonbrun J, Baker D (2006) Multipass membrane protein structure prediction using Rosetta. Proteins 62:1010-1025. CrossRef Medline

Yarov-Yarovoy V, DeCaen PG, Westenbroek RE, Pan CY, Scheuer T, Baker D, Catterall WA (2012) Structural basis for gating charge movement in the voltage sensor of a sodium channel. Proc Natl Acad Sci U S A 109: E93-E102. CrossRef Medline

Yokoi N, Fukata M, Fukata Y (2012) Chapter One—synaptic plasticity regulated by protein-protein interactions and posttranslational modifications. In: International review of cell and molecular biology (Kwang WJ, ed), pp 1-43. New York: Academic.

Yount JS, Moltedo B, Yang YY, Charron G, Moran TM, López CB, Hang HC (2010) Palmitoylome profiling reveals S-palmitoylation-dependent antiviral activity of IFITM3. Nat Chem Biol 6:610-614. CrossRef Medline 